\title{
Pseudo-Meigs syndrome in association with primitive peritoneal tumor*
}

\author{
Auriane De Neef ${ }^{1 \#}$, Jean-Christophe Noel ${ }^{2}$, Frédéric Buxant ${ }^{3}$ \\ ${ }^{1}$ Université Libre de Bruxelles, Brussels, Belgium \\ ${ }^{2}$ Department of Pathology, Erasme Hospital, Université Libre de Bruxelles, Brussels, Belgium \\ ${ }^{3}$ Department of Gynaecology, Iris Sud Hospital, Université Libre de Bruxelles, Brussels, Belgium \\ Email: "
}

Received 12 March 2012; revised 15 April 2012; accepted 26 April 2012

\begin{abstract}
We report the case of a 61-year old woman presented with a voluminous centro-pelvic mass $(22 \times 17 \mathrm{~cm})$, hydrothorax and ascites. Serum CA-125 was high $(11,000 \mathrm{UI} / \mathrm{ml})$. An explorative laparotomy located the mass between the rectum and the posterior wall of the uterus. The histological examination showed a primitive peritoneal tumor with serous and endometrioid differentiations. There was a left ovarian metastasis and both peritoneal and pleural liquids revealed no sign of malignancy. After the incomplete surgery, the hydrothorax and ascites decreased considerably. This association of Pseudo-Meigs' syndrome with primitive peritoneal tumor is uncommon. To our knowledge this is the first case described through a Medline search.
\end{abstract}

Keywords: Ovarian and Tubal Neoplasms; Peritoneal Neoplasm

\section{INTRODUCTION}

Pseudo-Meigs' syndrome is the association of an ovarian tumor with benign ascites and hydrothorax. The ovarian tumors include all benign ovarian tumors, other than ovarian fibromas, and all malignant ovarian tumors, either primitive tumor or metastasis from mostly gastrointestinal cancers. An important criterion is that the effusions have to disappear after the removal of the tumor.

In this case report, we present the association of Pseudo-Meigs' syndrome with primitive peritoneal tumor which has not previously been described. We will also review the different etiologies and the physiopathology of Pseudo-Meigs’ syndrome.

"Conflict of interest: all authors disclose any financial and personal relationships with other people or organizations that could inappropriately influence their work.

\#Corresponding author.

\section{CASE REPORT}

In June 2010, a 61-year old female patient was sent to us with suspicion of VIN3. She had had her menopause at the age of 55 and she was not taking any hormonal substitutive treatment. She was receiving treatment for diabetes and hypertension, both were stable.

On vulvar resection, the pathologist found a moderately differentiated microinvasive epidermoid carcinoma without extension. This was classified pT1bNx and the decision was a strict follow-up.

Seven months later, the patient related breathlessness. A right hydrothorax was found at the imagery (Figure 1) and also a large centro-pelvic mass $(22 \times 17 \mathrm{~cm})$ that seemed to come from the ovaries (Figure 2), with a left inguinal adenopathy $(2 \times 3 \mathrm{~cm})$ and a left renal adenopathy $(15 \mathrm{~mm})$. The pleural liquid was exsudative and no malignant cells were found. At the biology, there was no inflammatory syndrome and the serum CA 125 was high (11,000 UI/ml). The CEA was negative. An explorative laparotomy was then carried out and revealed a heterogeneous cystic centro-pelvic mass located between the rectum and the posterior wall of the uterus and attached

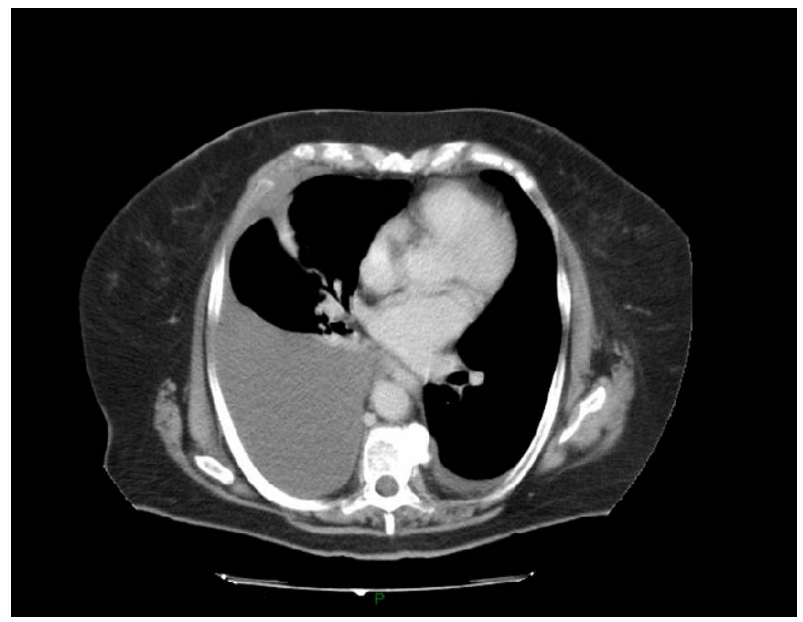

Figure 1. Thoracic CT scanner: massive right hydrothorax. 


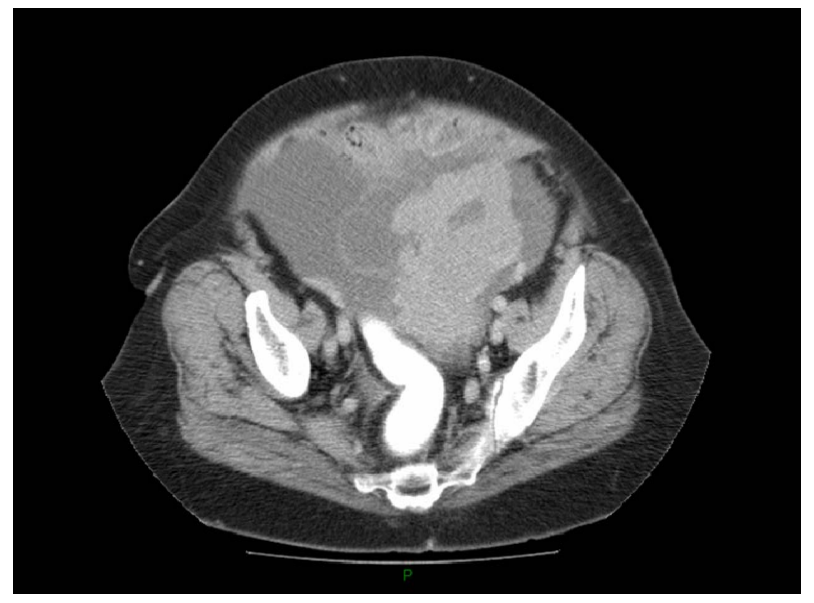

Figure 2. Abdominal CT scanner: heterogeneous centro-pelvic mass $(22 \times 17 \mathrm{~cm})$.

to the digestive peritoneum. This mass and the left ovary were resected and biopsies of both lateral paracolic gutters and endometrium were performed. The per-operative analysis of the mass wasn't able to differentiate an ovarian, peritoneal or digestive tumor so that no digestive resection was performed. There were no malignant cells in the peritoneal cytology. A complete histology showed a primitive peritoneal carcinoma with patterns of serous (positive CA 125) and endometrioid (positive CA 19.9) differentiations. The pathology of the left ovary revealed invasive implants of the peritoneal carcinoma with lymphovascular emboles. There was no more extension.

During the same surgery, a left inguinal adenopathy was resected and showed a metastasis of the previous vulvar carcinoma. Six days after surgery, the hydrothorax and the serum CA 125 decreased considerably (CA 125 at $2970 \mathrm{U} / \mathrm{ml}$ and CA 15.3 at $56 \mathrm{U} / \mathrm{ml}$ ). Because of the multiplicity of the lesions, a PET CT was performed. It revealed the rest of the centro-pelvic mass and other hypermetabolic lesions: para-aortic (L2) retroperitoneal adenopathies. A discrete pleural effusion remained. The therapeutic proposal was to begin with 6 cures of chemotherapy (Carboplatine $750 \mathrm{mg}$ - Paclitaxel $280 \mathrm{mg}$ ) followed by a second larger surgery. The pleural and peritoneal effusion completely disappeared after three cures and after the sixth cure, the CA-125 had become negative (CA 125 at $14 \mathrm{U} / \mathrm{ml}$ ). The second surgical intervention was then performed and failed to reveal any macroscopic lesions. The debulcking surgery consisted in hysterectomy, right annexectomy, pelvic lymphadenectomy and omentectomy. The pathology only showed a small residual tumor on left utero-sacral ligament, the debulcking was optimal. There was no need of adjuvant therapy and the decision was a strict follow-up.

\section{DISCUSSION}

The association of ovarian tumor, hydrothorax and as- cites were first described in 1887 by Demon [1]. In 1937, Meigs rediscovered this association and limited it to the ovarian fibromas [2] whereas Demon included all benign ovarian tumors and fibromas of the broad ligament. However, the essential elements of Meigs' and Demon's syndrome were that the effusions had to disappear after the removal of the tumor and that the effusions had to have strict characteristics (sterile cultures and negative cytology) [3].

Many cases of malignant tumors have been described in association with benign chest and abdominal effusions. The term Pseudo-Meigs' syndrome includes all benign (other than fibromas) and malignant ovarian tumors or ovarian metastasis [3]. They are mostly from gastro-intestinal (colo-rectal and gastric) cancers [4] but also from breast [5], renal cancers [6] and peritoneal gliomatosis [7]. The syndrome has also been reported in a few cases of leiomyomas of the uterus [8] and of the broad ligament [9].

The aetiology of the effusion is unknown but many hypotheses have been presented: the liquid can be either exudative or transudative. The main explanation for the transudation is that the pressure on the lymphatics in the tumor causes the fluid to escape through the superficial lymphatic vessels of the tumor. The ascitic fluid is then transferred to pleural space via transdiaphragmatic lymphatic channels. A hypothesis for exudation is that mediators produced by the tumor lead to increased vascular permeability. Another is that the peritoneal irritation by the tumor stimulates the production of peritoneal fluid [10].

In the present case, the patient presented a centro-pelvic mass and a small ovarian metastasis from primitive peritoneal tumor with a large pleural effusion and small amount of ascites. The diagnostic of Pseudo-Meigs syndrome is uncertain. Indeed, no malignant cells were found at the cytology but a pleural biopsy wasn't performed to confirm the benignity of the effusion. The fact that hydrothorax and ascites decreased considerably after the first incomplete surgery suggests that this is indeed a case of Pseudo-Meigs' syndrome.

The association of Pseudo-Meigs' syndrome with primitive peritoneal tumor is rare. To our knowledge this is the first case described.

\section{REFERENCES}

[1] Demons, A. (1887) Epanchements pleurétiques compliquant les kystes de l'ovaire. Bulletin et Memoires de la Societe des Chirurgiens Paris, 13, 771-776.

[2] Meigs, J.V. and Cass, J. (1937) Fibroma of ovary with ascites and hydrothorax. American Journal of Obstetrics \& Gynecology, 33, 249-267.

[3] Brun, J.L. (2007) Demons syndrome revisited: A review 
of the literature. Gynecologic Oncology, 105, 796-800. doi:10.1016/j.ygyno.2007.01.050

[4] Feldman, E.D., Hughes, M.S., Stratton, P., Schrump, D.S. and Alexander, H.R. Jr. (2004) Pseudo-Meigs' syndrome secondary to isolated colorectal metastasis to ovary: A case report and review of the literature. Gynecologic Oncology, 93, 248-251. doi:10.1016/j.ygyno.2003.12.025

[5] Fujii, M., Okino, M., Fujioka, K., Yamashita, K. and Hamano, K. (2006) Pseudo-Meigs' syndrome caused by breast cancer metastasis to both ovaries. Breast Cancer, 13, 344348. doi:10.2325/jbcs.13.344

[6] Tessmer, C.S., Barcellos, F.C. and Falchi, L.C. (2005) Pseudo-Meigs' syndrome associated to renal pelvis tumor. International Brazilian Journal Urology, 31, 256-258. doi:10.1590/S1677-55382005000300010

[7] Khan, J., McClennan, B.L., Qureshi, S., Martell, M., Lyer, A. and Bokhari, S.J. (2005) Meigs syndrome and gliomatosis peritonei: A case report and review of literature.
Gynecologic Oncology, 98, 313-317. doi:10.1016/j.ygyno.2005.03.048

[8] Migishima, F., Jobo, T., Hata, H., Sato, R., Ikeda, Y., Arai, M. et al. (2000) Uterine leiomyoma causing massive ascites and left pleural effusion with elevated CA 125: A case report. Journal of Obstetrics and Gynecology Research, 26, 283-287. doi:10.1111/j.1447-0756.2000.tb01323.x

[9] Buckshee, K., Dhond, A.J., Mittal, S. and Bose, S. (1990) Pseudo-Meigs' syndrome secondary to broad ligament leiomyoma: A case report. Asia-Oceania Journal of $\mathrm{Ob}$ stetrics and Gynecology, 16, 201-205. doi:10.1111/j.1447-0756.1990.tb00226.x

[10] Rubinstein, Y., Dashkovsky, I., Cozacov, C. and Hadary, A. (2009) Pseudomeigs' syndrome secondary to colorectal adenocarcinoma metastasis to the ovaries. Journal of Clinical Oncology, 27, 1334-1336. doi:10.1200/JCO.2008.20.1756 